\title{
Assessing Food Insecurity in Medical Students
}

Mary M. Flynn, PhD, RD, LDN; Kristina Monteiro, PhD; Paul George, MD, MHPE;

Allan R. Tunkel, MD, PhD

BACKGROUND AND OBJECTIVES: Food insecurity, defined as the lack of reliable access to sufficient quantities of affordable, nutritious food and present in $11.1 \%$ of the general population, has not been assessed in medical students. Food insecurity is related to adverse outcomes for both health and academics in undergraduate students. Assessing the presence of food insecurity in medical students, a population at high risk for burnout and depression, may allow for intervention in order to improve overall wellness in this population. The objectives of this study were to assess the prevalence of food insecurity in a medical student population and identify potential reasons for any measured food insecurity.

METHODS: We used a questionnaire that included the US Household Food Security Survey Module: Six-Item Short Form. The survey was emailed to all students enrolled at a Northeastern medical school $(\mathrm{N}=588)$ to assess food insecurity in the previous 3 months. The questionnaire included potential reasons for food insecurity.

RESULTS: The survey response rate was $22.4 \%$. Food insecurity was present in $11.8 \%$ of these respondents. The main reasons selected for food insecurity were not being able to get to the store (33.9\%), followed by insufficient funds $(30.4 \%)$.

CONCLUSIONS: Prevalence of food insecurity in medical students may be similar to the general population. While the results of this study represent only one medical school, we believe these results are similar at other medical schools. Interventions to reduce food insecurity in this population are necessary to improve overall student wellness.

(Fam Med. 2020;52(7):512-3.)

doi: 10.22454/FamMed.2020.722238

5 ood insecurity, defined as the lack of reliable access to sufficient quantities of affordable, nutritious food, ${ }^{1}$ has been assessed in undergraduates at US colleges in multiple studies. ${ }^{2}$ Food insecurity is related to poorer health and adverse academic outcomes. ${ }^{3}$ In a previous study, one author (M.F.) initiated a 4-week cooking program at the Warren Alpert Medical School of Brown University (AMS) that was offered Form. ${ }^{5}$ broadly. Based on the impact seen in undergraduates who are food insecure, it is reasonable to identify if food insecurity is present in medical students. Medical students are at a high risk for burnout and depression. Food insecurity, if prevalent in medical students, may contribute to risk of medical students' poorer wellness status. The objective of this research was to assess the prevalence of food insecurity of all enrolled students at the AMS.

\section{Methods}

We sent a Qualtrics questionnaire by email in March 2019 to all students enrolled at the Warren Alpert Medical School of Brown University $(n=588)$ that included demographic questions, amount of school loan for current year, and the US Household Food Security Survey Module: Six-Item Short Form ${ }^{5}$ assessing food security for the previous 3 months. The survey was sent three times in a 30-day period. The food insecurity scores were categorized as high food security (score 0), marginal food security (score 1) low food security (score 2 to 4), and very low food security (score 5 to 6 ), based upon USDA guidelines. ${ }^{5}$ Four questions were also included that assessed potential reasons for food insecurity. The principle by the US Household Food Security Survey Module: Six-Item Short

To our knowledge, food insecurity has not been assessed in students enrolled in medical schools more
From the Miriam Hospital and Brown University, Providence, RI (Dr Flynn); and the Warren Alpert Medical School, Brown University, Providence, RI (Drs Monteiro, George, and Tunkel). 
investigator's institutional review board deemed this research exempt.

\section{Results}

One hundred thirty-two students $(22.4 \%)$ responded to the survey. Sixty-seven percent were male, and age was $26 \pm 2.56$ years. Regarding ethnicity, $86.9 \%$ reported non-Hispanic; for race, $65 \%$ white, $6.8 \%$ black or African American, 18\% Asian, and the remaining being "other." For the question "Was there ever a time in medical school when you did not have enough food," $25.7 \%$ responded yes. Food insecurity was prevalent in $11.8 \%$ with a mean score of 4.0 \pm 1.5 (maximum score of 6.0 ), which is the upper limit for a "low food security" score. The potential reasons selected by respondents for food insecurity are shown in Table 1 . Nearly $60 \%$ reported having a student loan with a mean loan of $\$ 54,718$, compared to $61 \%$ of the overall students at AMS reporting having a student loan with a mean loan of $\$ 47,189$. There was no relationship of student loan amount to food insecurity $(r=.15 ; P=.10)$.

\section{Discussion}

In this survey of medical students, food insecurity prevalence was lower than what was reported in our initial study, but was comparable to the prevalence of $11.1 \%$ found in the general US population. ${ }^{6}$ The mean score of $4.0 \pm 1.5$, however, was higher than the mean score of $2.6 \pm 1.6$ found in the initial study. ${ }^{7}$ The higher percentage found in the earlier protocol may have represented students who chose to participate in the cooking program due to food insecurity; however, the study announcement did not include wording that the program was attempting to measure and address this construct.

The main limitation of this study is the relatively low response rate. However, any measured food insecurity has the potential to adversely affect a medical student. The results of this study and our previous work indicate that food insecurity cannot be ignored in the medical student population. Further studies examining food insecurity across institutions and across training levels (in both undergraduate medical education and graduate medical education) are needed. The implications of food insecurity in medical trainees are significant, as these learners are already at an extraordinarily high risk of burnout and depression.

There are strategies to reduce food insecurity within the medical student population. Of the four potential reasons for being food insecure provided in the survey, the one that received the most responses was not being able to get to a store that sold the food the student wanted. AMS is located in a food desert, which the USDA defines as no supermarket with nutritious foods within 0.5 to 1.0 miles,${ }^{8}$ as the average distance to a supermarket is $2.2 \pm 0.4$ miles, which could account for the response. In addition, factors contributing to burnout in medical school (such as licensing examinations, the increased competition of the Match) may also decrease the ability of medical students to purchase food, making them food insecure.

To our knowledge, this study is the first of others that need to be done to truly measure the impact of food insecurity in medical students.

Table 1: Reasons Selected for Food Insecurity

\begin{tabular}{|l|l|}
\hline \multicolumn{1}{|c|}{ Reason } & \multicolumn{1}{|c|}{$\begin{array}{c}\text { Percent } \\
\text { Responding }\end{array}$} \\
\hline I did not have sufficient money for food. & 30.49 \\
\hline I was not able to get to a store that sold the food I wanted. & 33.96 \\
\hline I did not have enough time to grocery shop. & 30.18 \\
\hline $\begin{array}{l}\text { I am not able to cook for myself (do not know how; do not have } \\
\text { cookware, etc). }\end{array}$ & 23.81 \\
\hline
\end{tabular}

Interventions to decrease food insecurity in medical students should also be studied, including the reduction of loan debt, increasing access to being able to purchase food (through online services such as Amazon Prime, for example) and by decreasing factors that increase burnout. Studies looking at food insecurity in other learners, such as residents should also be examined. Food insecurity among medical students is an issue that deserves further attention moving forward and we hope that this study leads to other medical schools examining food insecurity in their students.

CORRESPONDING AUTHOR: Address correspondence to Dr Mary M. Flynn, The Miriam Hospital and Brown University, 164 Summit Ave, Providence, RI 02906. 401-793-4707. Mary_Flynn@brown.edu.

\section{References}

1. Nord M, Coleman-Jensen A, Andrews M, Carlson S. Household food security in the United States, 2009. Washington, DC: USDA Economic Research Service; 2010. Accessed March 14, 2017.

2. Nazmi A, Martinez S, Byrd A, et al. A systemic review of food security among US students in higher education. J Hunger Environ Nutr. $2019 ; 14: 5,725-740$

3. Bruening M, Argo K, Payne-Sturges D, Laska MN. The Struggle Is Real: A Systematic Review of Food Insecurity on Postsecondary Education Campuses. J Acad Nutr Diet. 2017;117(11):1767-1791

4. Flynn MM, George P, Schiffman FJ. Food is medicine: using a 4-week cooking program of plant-based, olive oil recipes to improve diet and nutrition knowledge in medical students. Med Sci Educ. 2019;29(1):61-66.

5. US Department of Agriculture Economic Research Service. U.S. Household food security module: six-item short form. Washington, DC: USDA; 2012. https:/www.ers.usda.gov/topics/ food-nutrition-assistance/food-security-in-theus/survey-tools?\#six Accessed December 1, 2016

6. US Department of Agriculture Economic Research Service. Food Security in the U.S. Washington, DC: USDA; 2019. https://www. ers.usda.gov/topics/food-nutrition-assistance/ food-security-in-the-us/. Accessed December 1, 2019.

7. Flynn MM, George P, Schiffman FJ. Improving Food Security in Medical Students Using a Cooking Program. J Hunger Environ Nutr. 2019;1-9.

8. Rhone A. VPM, Dicken C, Williams R, Breneman V. Low-income and low-supermarketaccess census tracts, 2010-2015. Washington, DC: USDA; 2017. 